\title{
Quasi-Free Scattering from Relativistic Carbon and Oxygen Isotopes
}

\author{
Matthias Holl*, Leyla Atar and Valerii Panin \\ Institut für Kernphysik, Technische Universität Darmstadt \\ E-mail: mholleikp.tu-darmstadt.de
}

Single nucleon knockout and quasi-free scattering reactions are valuable tools to study singleparticle properties of nuclei. Particularly, it has been argued, that they can be used to study spectroscopic factors on an absolute scale. Quenching of these spectroscopic factors as compared to shell-model predictions has been observed in nuclear knockout reactions. While for stable isotopes these findings are in agreement with results obtained in quasi-free electron scattering, a surprisingly large dependancy of this quenching on the neutron-proton asymmetry has been observed, motivating further studies using quasi-free proton scattering.

Quasi-free scattering of both stable and exotic light nuclei has been studied in inverse kinematics at GSI. While in a first protoype experiment a $12 \mathrm{C}$ beam was accelerated to $500 \mathrm{AMeV}$ by the SIS18 heavy ion synchrotron, in a second experiment mixed secondary beams created by impinging $40 \mathrm{Ar}$ on a production target at the entrance of the fragment separator FRS were used. In both cases, the incoming beam as well as all reaction products were detected in kinematically complete measurements at the R3B-LAND setup.

Results for cross sections, spectroscopic factors and momentum distributions will be shown for different carbon and oxygen isotopes and compared to results obtained for knockout reactions as well as DWIA-calculations. Furthermore, excitation spectra of the reaction products will be discussed.

53rd International Winter Meeting on Nuclear Physics

26-30 January 2015

Bormio, Italy

\footnotetext{
*Speaker.
} 
Investigating the influence of long- and short-range correlations between nucleons on the structure of nuclei is one of the major topic of modern nuclear physics. These correlations influence the occupancies of nuclear shells and lead to partially occupied orbits [1]. To quantify this, often the spectroscopic factor $S_{j}$ is used, which is defined via the overlap between the wavefunction of the initial and final state of a nuclear reaction. In the case of the removal of a single nucleon from a nucleus with $A$ nucleons this can be written as an expansion in single particle terms,

$$
\left\langle\Psi_{A-1}(\vec{r}) \mid \Psi_{A}(\vec{r})\right\rangle=\sum_{j=I_{A}-I_{A-1}}^{I_{A}+I_{A-1}} c_{j} \psi_{j}(\vec{r}) .
$$

With the single-particle states normalized to unity, the spectroscopic factor can be defined as $S_{j}=\left|c_{j}\right|^{2}$. With the spectroscopic factor and the single-particle cross section $\sigma_{s p}$, the reaction cross section can be written in the factorised form

$$
\sigma_{t h}=\sum_{j} S_{j} \cdot \sigma_{s p}(n l j) .
$$

In quasi-free electron scattering (e,e'p) reactions on stable isotopes, a reduction to $60-70 \%$ of the independent particle model value was found [2]. Over the past two decades, knockout reactions in inverse kinematics have been developed into a valuable tool for the study of single particle properties nuclei far away from stability. This has made it possible to extend the studies of quenching to exotic nuclei [3-5], confirming the findings from (e,e'p) and additionially suggesting a dependency of this quenching on isospin asymmetry [4]. However, this dependency is contested since it was not observed in several transfer reaction experiments [6]. In addition, ab-initio calculations, including short-range and partially long-range correlations, predict a less pronounced dependence on isopspin asymmetry as experimentally observed $[1,7]$.

A major constrain for both knockout and transfer reactions is their strong surface localization due to absorption in the nuclear medium. To overcome this problem, the application of quasi-free proton scattering to exotic nuclei has been proposed. In such reactions, a proton of a typical energy of $100-1000 \mathrm{MeV}$ is scattered off a nucleus. In an ideal case, a proton with momentum $\vec{p}_{p}$ is incident on a nucleon which is moving inside a nucleus with momentum $\vec{p}_{N}$ and no further collisions occur. Therefore, both the scattered proton and the knocked-out nucleon leave the nucleus with momentum $\vec{p}_{p^{\prime}}, \vec{p}_{N^{\prime}}$ while the remaining nucleus receives a recoil momentum $\vec{p}_{A-1}=-\vec{p}_{N}$. Momentum conservation then gives

$$
\vec{p}_{p}=\vec{p}_{p^{\prime}}+\vec{p}_{N^{\prime}}+\vec{p}_{A-1}
$$

Since nuclear absorption plays a much smaller role for energetic nucleons, such a case is not unlikely. Quasi-free scattering (QFS) offers therefore almost unique possiblities to study both inner and valence shells [8].

The knocked-out nucleon leaves a single-particle hole in the nucleus which decays depending on the the excitation energy of the residual nucleus $E_{A-1}^{*}$. If the excitation energy is lower than the binding energy of the least bound nucleus $S_{N}$ the nucleus will deexcite via $\gamma$-emission, while for $E_{A-1}^{*}>S_{N}$ a breakup of the residual nucleus is more likely. It is therefore possible to obtain information on the shell the nucleon was removed from in two ways, by measuring momentum and 


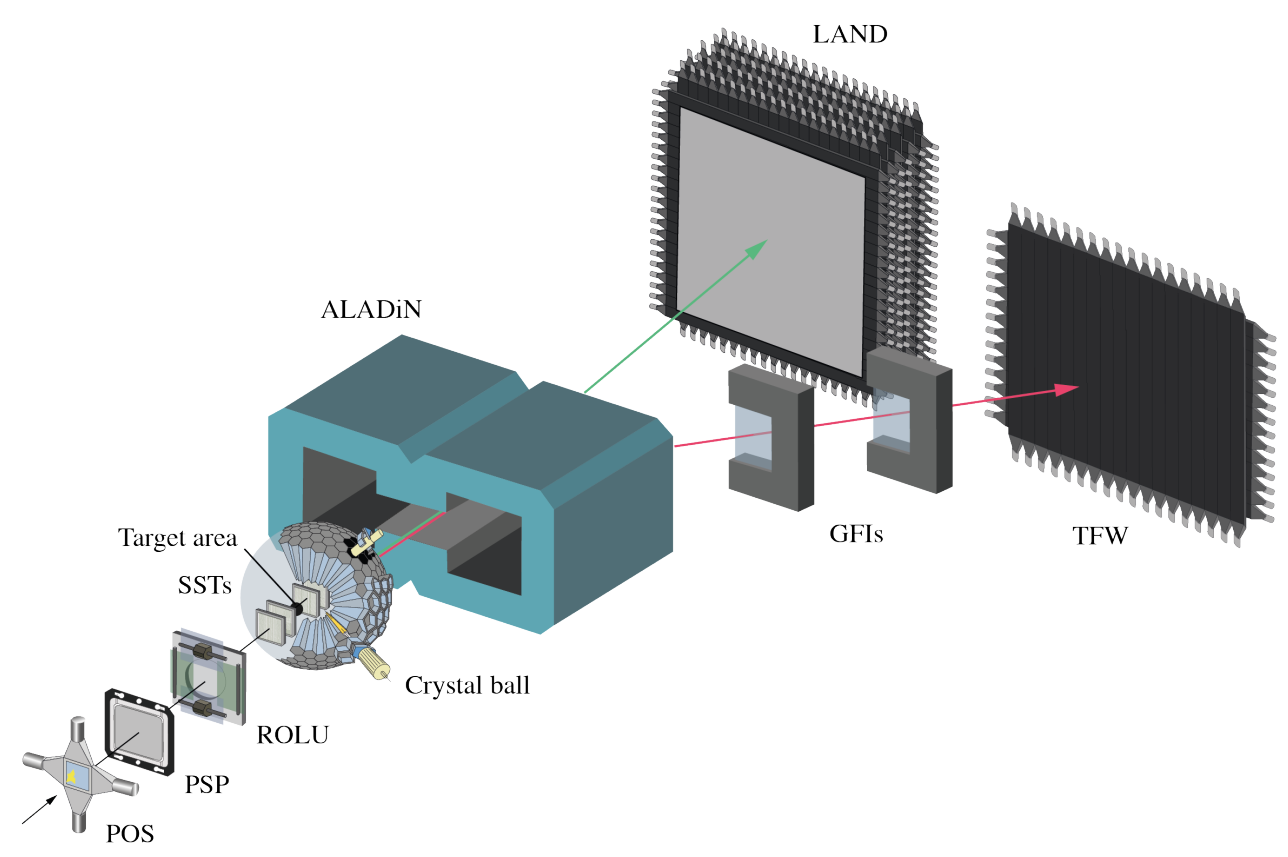

Figure 1: Schematic of the $\mathrm{R}^{3} \mathrm{~B}$-LAND setup at GSI. The setup allows to measure reactions with relativistic radioactive beams in complete kinematics. The target is surrounded eight double-sided silicon strip detectors (SSTs), four of which are mounted as a box around the target (not shown). The proton branch of the setup, consisting of two drift chambers and a second time-of-flight wall, is not shown for simplicity. Image taken from [12].

excitation of the remaining nucleus and by measuring the momenta of the scattered nucleons.

A commonly used approach for the description of quasi-free scattering is the distorted wave impulse approximation (DWIA) $[9,10]$ which assumes a single elastic interaction between the incident and the knocked-out nucleon and incorporates effects like multiple scattering or absorption only by using single-nucleon wave functions distorted by a complex optical potential[11].

Figure 1 shows the $\mathrm{R}^{3} \mathrm{~B}$-LAND setup as it was used in August 2010. The setup provides the possibility to measure quasi-free scattering reactions with relativistic radioactive beams in complete kinematics. The beams were provided by the UNILAC ${ }^{1}$ and injected into the SIS-18 ${ }^{2}$ [13]. After the acceleration, the ions can either be directly transported to the experimental area or be used to create a wide range of nuclei in fragmentation reactions. These secondary beams are then delivered to the experiment by the $\mathrm{FRS}^{3}$ [14]. In this case inccoming nuclei are identified using their charge and time-of-flight. The charge was determined using a position-senstive pin diode (PSP) while for

\footnotetext{
${ }^{1}$ UNIversal Linear ACcelerator

${ }^{2}$ SchwerIonenSynchrotron, Heavy Ion Synchrotron

${ }^{3}$ FRagment Separator
} 


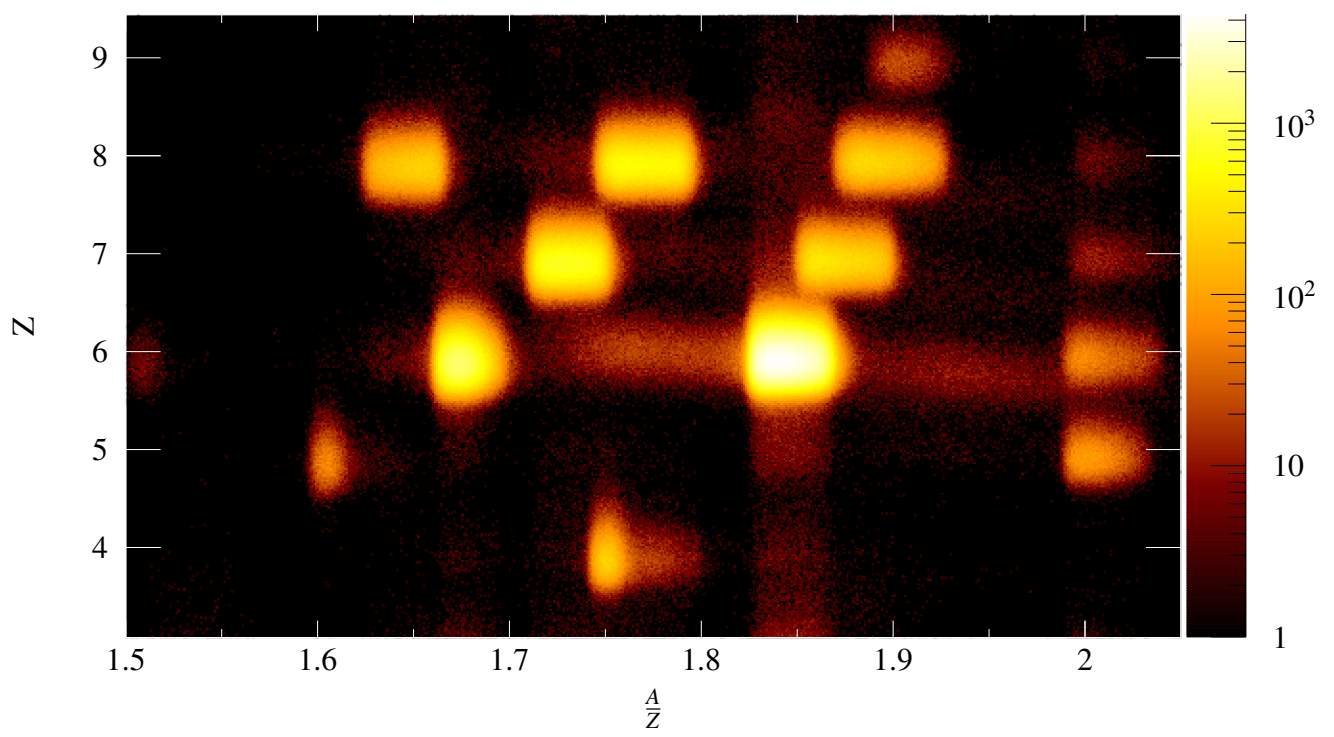

Figure 2: Identification plot for the incoming nuclei. The charge of the nuclei is plotted against their mass-to-charge ratio.

the time-of-flight measurement two plastic scintillators, one at the focal plane S8 of the FRS and one at the entrance of Cave $\mathrm{C}$, were used. From the time-of-flight and the magnetic rigidity $B \rho$ of the FRS, the mass-to-charge ratio $(A / Z)$ can be calculated using the relation

$$
B \rho=\frac{A}{Z} \beta \gamma
$$

Figure 2 shows the charge of the nuclei as a function of $\frac{A}{Z}$ for light nuclei with a charge up to $Z=9$ and $A / Z=2.1$

The target was surrounde by eight DSSDs, mounted in close proximity to the target, two in front of the target, two behind it, and the remaining for arranged as a box around the target. The target chamber was surrounded by the Crystal Ball, a $4 \pi$ array consisting of 162 sodium iodide crystals [15], used to detect $\gamma$-rays emitted by the deexciting fragment and the protons from (p,2p). The reaction products passed then through the magnetic field of the ALADIN dipole magnet which bends the flight path of fragments and protons, while neutrons are not affected. The heavy fragments are bent towards the fragment arm of the setup, consisting of two fiber detectors and a time-of-flight wall.

In a similar manner to the icoming nuclei, the fragments produced in the reactions are identified by their charge and mass. The charge is determined in the DSSD directly behind the target and a time-of-flight wall at the end of the setup. This redundancy allow to distinguish between charge changing reactions in the target and breakup behind the target. Figure 3 shows the fragment charge identification for the case of incoming ${ }^{11} \mathrm{C}$. The energy loss in the time-of-flight wall is shown as a function of the energy loss in the DSSD directly behind the target. All charges up to $Z=6$ can be identified.

Mass and momentum of the outgoing particles are calculated by a tracking algorithm which uses the measured positions from the DSSDs in front of the magnet and two fiber detectors behind it, 


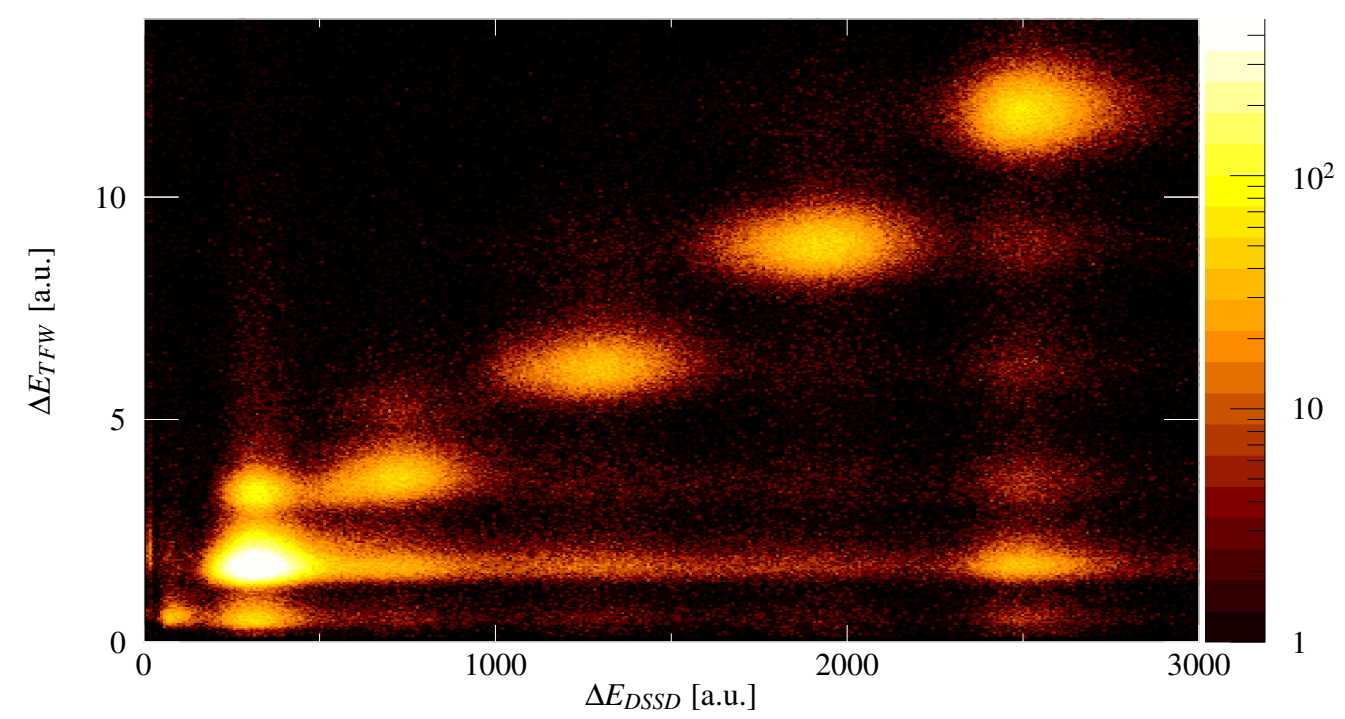

Figure 3: Charge identification of the fragments behind the target. The energy loss in the time-offlight wall at the end of the setup is plotted against the energy loss in the DSSD directly behind the target.

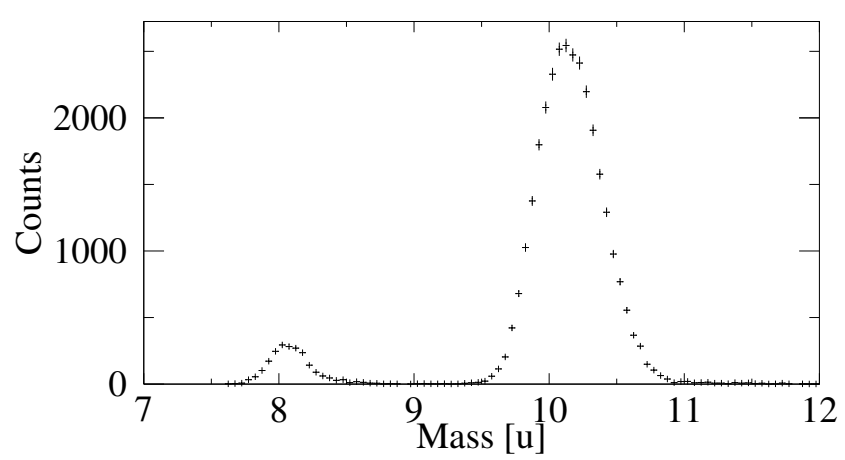

Figure 4: Mass spectrum for incoming ${ }^{11} \mathrm{C}$ and outgoing Boron, measured in coincidence with a proton detected in the Crystal Ball.

together with the time-of-flight measured by the ToF-wall to reconstruct the path the particles took through the magnetic field of ALADIN. Figure 4 shows the mass spectrum of Boron isotopes measured in coincidence with two protons in the Crystal Ball.

The setup also offers the possibility to detect and track fast neutrons and protons which are emitted from a deexciting fragment. The neutrons, unaffected by the magnetic field, fly straight into LAND [16]. The protons are bent in the magnetic field towards the proton branch of the setup which consists of two proton drift chambers and a second time-of-flight wall.

The experimental cross section can be calculated using

$$
\sigma=\frac{N_{r}}{N_{t} \cdot N_{i} / \varepsilon}
$$

with the number reactions $N_{r}$, the number of nuclei incident on the target, $N_{i}$ and the number of target atoms, $N_{t}$. Due to the low reaction probability, $N_{i}$ can be approximated by the number of 
unreacted nuclei. The efficiency correction $\varepsilon$ contains therefore only the probability of the Crystal Ball to detect the two protons from a $(\mathrm{p}, 2)$ reaction. This probability was obtained from Geant 3 simulations using the r3broot framework [17] and a QFS kinematics code developed by L. Chulkov [18].

To obtain the cross section for quasi-free scattering on hydrogen, measurements with two targets were done, a polyethylene $\left(\mathrm{CH}_{2}\right)$ and a carbon target. The experimental runs with the carbon target were used to subtract the contribution by the carbon in $\mathrm{CH}_{2}$ and to determine the background, and the quasi-free scattering cross section was obtained using

$$
\sigma_{Q F S}=\frac{1}{2}\left(\sigma_{C H_{2}}-\sigma_{C}\right),
$$

A prototype experiment for $(\mathrm{p}, 2 \mathrm{p})$-reactions in inverse kinematics was performed using ${ }^{12} \mathrm{C}$. The results are in good agreement with both (e,e'p) and (p,2p) measurements performed in direct kinematics. Approximately $65 \%$ of the theoretically expected spectroscopic strength was observed. Most of the strength was found in the ground state, while highly excited states due to knockout from the deeply bound s-shell were also observed [19].

Applying ( $\mathrm{p}, 2 \mathrm{p}$ ) to unstable isotopes, in the case of the quasi-free proton knockout from ${ }^{11} \mathrm{C}$, a cross section of $\sigma=17.3(8) \mathrm{mb}$ was found [20]. DWIA calculations assuming a knockout from the pshell give a cross section of $\sigma_{t h}=32.0 \mathrm{mb}$ [21], leading to a spectroscopic factor of $S=2.16(10)$. Momentum distributions obtained in these calculations have been compared to the experimental data and show good agreement.

The population of excited states below the particle threshold was determined by fitting responses to single excited states simulated using Geant 3 and $\mathrm{r} 3$ broot to the experimental spectrum. The overall population of excited states is high $(\approx 50 \%)$, indicating a strong contribution of particle-hole states to the ground state of ${ }^{11} \mathrm{C}$. This is also in agreement with the observation of large cross sections for the population of low-lying unbound states.

A similar analysis for the isotopes of the oxygen chain is currently ongoing. The investigation of both quasi-free proton and neutron knockout will be one of the main areas of activity at the future $\mathrm{R}^{3} \mathrm{~B}$ setup at FAIR.

This work was supported by the BMBF, the State of Hesse (LOEWE Centre HIC for FAIR), and through the GSI-TU Darmstadt cooperation agreement.

\section{References}

[1] W.H. Dickhoff and C. Barbieri, Self-consistent Green's function method for nuclei and nuclear matter, Progress in Particle and Nuclear Physics 52, 377-496, 2004.

[2] G.J. Kramer, H.P. Blok, and L. Lapikás, A consistent analysis of (e,ep) and (d,3He) experiments, Nuclear Physics A 679, 267-286, 2001.

[3] P.G. Hansen and J.A. Tostevin, Direct Reactions With Exotic Nuclei, Annual Review of Nuclear and Particle Science 53, 219-261, 2003.

[4] A. Gade et al., Reduction of spectroscopic strength: Weakly-bound and strongly-bound singleparticle states studied using one-nucleon knockout reactions, Physical Review C 77, 044306, 2008. 
[5] J. Enders et al., Spectroscopic factors measured in inclusive proton-knockout reactions on $8 B$ and 9 C at intermediate energies, Physical Review C 67, 064301, 2003.

[6] J. Lee et al., Neutron spectroscopic factors of 34Ar and 46Ar from (p,d) transfer reactions, Physical Review C 83, 014606, 2011.

[7] G. F. Grinyer et al., Knockout Reactions from p-Shell Nuclei: Tests of Ab Initio Structure Models, Physical Review Letters 106, 162502, 2011.

[8] G. Jacob and T.A.J. Maris, Quasi-Free Scattering and Nuclear Structure, Reviews of Modern Physics 38, 121-142, 1966.

[9] N.S. Chant and P.G. Roos, Distorted-wave impulse-approximation calculations for quasifree cluster knockout reactions, Physical Review C 15, 57-68, 1977.

[10] N.S. Chant and P.G. Roos, Spin orbit effects in quasifree knockout reactions, Physical Review C 27, 1060-1072, 1983.

[11] T. Aumann, C. A. Bertulani, and J. Ryckebusch, Quasifree ( $p, 2 p)$ and (p,pn) reactions with unstable nuclei, Physical Review C 88, 064610, 2013.

[12] S. Altstadt, 13,14B(n,g) via Coulomb Dissociation to Constrain the Astrophysical r-Process, Dissertation, Johann Wolfgang Goethe-Universität, Germany, 2014.

[13] K. Blasche, The heavy ion synchrotron SIS, in: EPAC 92: Third European Particle Accelerator Conference, 1992, 9-13.

[14] H. Geissel et al., The GSI projectile fragment separator (FRS): a versatile magnetic system for relativistic heavy ions, Nuclear Instruments and Methods in Physics Reseach B, 1992.

[15] V. Metag, D. Habs, and K. Helmer, The Darmstadt-Heidelberg-Crystal-Ball, in: Lecture Notes in Physics, 178. 1983, 163-178.

[16] Th. Blaich et al., A large area detector for high-energy neutrons, Nuclear Instruments and Methods in Physics Research Section A 314, 136-154, 1992.

[17] D. Bertini, R3BRoot, simulation and analysis framework for the R3B experiment at FAIR, Journal of Physics: Conference Series 331, 032036, 2011.

[18] L.V. Chulkov et al., Quasi-free scattering with 6,8He beams, Nuclear Physics A 759, 43-63, 2005.

[19] V. Panin, Fully Exclusive Measurements of Quasi-Free Single-Nucleon Knockout Reactions in Inverse Kinematics, Dissertation, Technische Universität Darmstadt, 2012.

[20] M. Holl, Quasi-Free Scattering from Relativistic Neutron-Deficient Carbon Isotopes, Dissertation, Technische Universität Darmstadt, 2014.

[21] C. Bertulani. private communication. 